\title{
Ethylene-Induced Vinblastine Accumulation Is Related to Activated Expression of Downstream TIA Pathway Genes in Catharanthus roseus
}

\author{
Xi Wang, ${ }^{1}$ Ya-Jie Pan, ${ }^{1}$ Bo-Wen Chang, ${ }^{2}$ Yan-Bo Hu, ${ }^{2}$ \\ Xiao-Rui Guo, ${ }^{1}$ and Zhong-Hua Tang ${ }^{1}$ \\ ${ }^{1}$ Key Laboratory of Forest Plant Ecology, Northeast Forestry University, Harbin 150040, China \\ ${ }^{2}$ College of Life Science, Northeast Forestry University, Harbin 150040, China
}

Correspondence should be addressed to Xiao-Rui Guo; xruiguo@nefu.edu.cn and Zhong-Hua Tang; tangzh@nefu.edu.cn

Received 13 December 2015; Revised 30 March 2016; Accepted 6 April 2016

Academic Editor: Sudhir Sopory

Copyright @ 2016 Xi Wang et al. This is an open access article distributed under the Creative Commons Attribution License, which permits unrestricted use, distribution, and reproduction in any medium, provided the original work is properly cited.

\begin{abstract}
We selected different concentrations of ethephon, to stress C. roseus. We used qRT-PCR and HPLC followed by PCA to obtain comprehensive profiling of the vinblastine biosynthesis in response to ethephon. Based on our findings, the results showed that the high concentration of ethephon had a positive effect at both transcriptional and metabolite level. Meanwhile, there was a remarkable decrease of hydrogen peroxide content and a promoted peroxidase activity in leaves. The loading plot combination with correlation analysis suggested that $C r \operatorname{Pr} x 1$ could be regarded as a positive regulator and interacts with ethylene response factor $(E R F)$ to play a key role in vinblastine content and peroxidase (POD) activity. This study provides the foundation for a better understanding of the regulation and accumulation of vinblastine in response to ethephon.
\end{abstract}

\section{Introduction}

Catharanthus roseus (L.) G. Don., also called Madagascar periwinkle, is widely used in studies as a model of medical plant for many kinds of terpenoid indole alkaloids (TIAs) [1]. Because of the antineoplastic activity in the treatment of many cancers, some of them have medicinal and scientific research value, such as vinblastine [2,3]. The early stages of vinblastine biosynthesis in $C$. roseus involve the formation of tryptamine from tryptophan and its condensation with secologanin to produce the central intermediate strictosidine, the common precursor for the monoterpenoid indole alkaloids, vindoline and catharanthine (Figure 1) [3]. These two monomeric alkaloids will then be enzymatically condensed to form the bisindole alkaloid vinblastine (Figure 1) [4].

Various proteins are involved in plant defense and secondary metabolic responses. Among these proteins, class III plant peroxidases (EC 1.11.1.7) are well known. As is reported, there are various abbreviations used for class III plant peroxidases such as POD, POX, and PRX [5]. Peroxidases (POD), always known as a type of antioxidant, are also involved in the biosynthesis of secondary metabolites for catalyzing production of vinblastine. Although the functions of POD are not well understood in plants, the enzyme has been recognized with medicinal properties [5]. The above statement is the case of the TIAs of Catharanthus roseus [6]. Sottomayor et al. found that peroxidase was purified to homogeneity and a channeling mechanism was proposed for the peroxidase mediated-vacuolar synthesis of $\alpha-3^{\prime}, 4^{\prime}$ anhydrovinblastine (AVLB) $[4,6]$. AVLB can be converted into vinblastine, which is biosynthesized through coupling of the monomeric precursors vindoline and catharanthine [7].

The POD encoded by $\mathrm{CrPrxl}$ gene is also a multifunctional enzyme that has another function of cleaning up the oxidation of small molecules including $\mathrm{H}_{2} \mathrm{O}_{2}$ in the cells. $\mathrm{H}_{2} \mathrm{O}_{2}$ is an electron-accepting substrate for a wide variety of peroxidase-dependent reactions; thus, POD is generally considered to be merely a ROS-detoxifying enzyme [8]. The breakdown of $\mathrm{H}_{2} \mathrm{O}_{2}$ accumulation by the POD reaction is highly active especially in the presence of ROS-scavenging POD substrates [9]. They have also been involved in secondary metabolism, in root elongation, and in hydrogen 


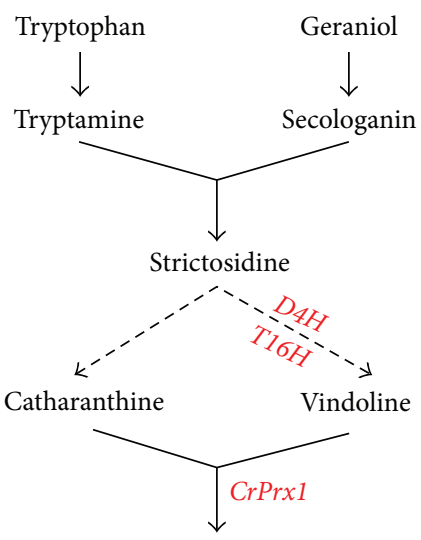

$\alpha-3^{\prime}, 4^{\prime}$-Anhydrovinblastine (AVLB)<smiles>[V][Tl]</smiles>

Vinblastine

FIGURE 1: The pathway of terpenoid indole alkaloids biosynthesis in Catharanthus roseus.

peroxide scavenging and production; furthermore, they are thought to play significant roles for plants in stress resistance and adaptation $[6,10-16]$. Although the detailed correlation between $\operatorname{CrPrx1}$ transcript and alkaloid levels was not clear, evidence further supported an important role for POD and CrPrxl in indole alkaloid biosynthesis, revealing the potential of $\operatorname{CrPr} x 1$ as a molecular tool for the manipulation of alkaloid metabolism. We have obtained several partial nucleotide sequences which were used to isolate the downstream TIA pathway genes (DAT, D4H, T16H, and CrPrx1) and an ethylene response factor gene $(E R F)$ by a RT-PCR to figure out the relationship between vinblastine biosynthesis and the expression of genes under ethylene-regulated control.

Plant secondary metabolites play critical roles in plantenvironment interactions. Thus, there are many environmental conditions that could frequently affect their synthesis, such as salinity, light, nitrate, and potassium [17-21]. Ethylene, known as a phytohormone, plays an important role in regulating plant growth and development among the whole life cycle of the plant [22-24]. Some research reported that the biotic or abiotic stress-induced expression of CrPrxl is conferred by the nature of the $5^{\prime}$ flanking regions of the genes that contain many kinds of potential stress-responsive cis-elements [25]. Physical injuries promoted an increase of production of nicotine which resulted from the transcriptional activation of the putrescine $N$-methyltransferase gene and this gene encodes a regulatory enzyme in nicotine biosynthesis [26, 27]. In this way, we mainly discuss the relationship between the accumulation of vinblastine and transcriptional level of $\operatorname{Cr} \operatorname{Pr} x 1$, which plays a putative role in catalyzing the condensation of vindoline and catharanthine.

\section{Materials and Methods}

2.1. Plant Materials and Cultivation Methods. C. roseus seedlings were germinated in perlite with distilled water until having grown out two pairs of leaves and then transferred to standalone plates with Hoagland nutrient solution. The seedlings were kept in a growth chamber at $28^{\circ} \mathrm{C}$ under a $16 \mathrm{~h}$ photoperiod.

2.2. Ethephon Treatment. Seedlings from the three-monthold plants were harvested, and 3 fully expanded leaves of seedlings were randomly selected and subjected to hydroponic treatment. For ethephon treatment, seedlings were cultivated in the Hoagland nutrient solution containing ethephon. We illustrated all the concentrations of ethephon used in our experiment as $45 \mu \mathrm{M}, 60 \mu \mathrm{M}$, and $100 \mu \mathrm{M}$ and the treatment lasted 4 days.

2.3. Ethylene Release Measurement. For measurement of ethylene released from grown plants, plants with or without ethephon treatment grew in a Bunsen beaker and the Bunsen beaker was sealed to air tightness with a plastic membrane. Ethylene that was released by treated plants was determined by gas chromatography (Agilent Technologies, 7890A GC Systems). One milliliter of air from each container was taken to detect the presence of ethylene.

2.4. Alkaloid Analysis. Dry leaf powder ( $0.3 \mathrm{~g})$ was dissolved in $10 \mathrm{~mL}$ absolute methanol (analytical grade) for extraction of vinblastine, vindoline, and catharanthine. Low-frequency ultrasonication $(250 \mathrm{~W}, 40 \mathrm{kHz})$ was used to extract the alkaloids for $20 \mathrm{~min}$. The methanol extract was centrifuged at $8000 \times \mathrm{g}$ for $10 \mathrm{~min}$, concentrated to $1 \mathrm{~mL}$, and analyzed by HPLC (Jasco, VG, England) equipped with a Waters ODS $\mathrm{C}_{18}$ reversed-phase column $(250 \times 4.6 \mathrm{~mm}, 5 \mu \mathrm{m})$ and a photodiode-array detector at $220 \mathrm{~nm}$. Sample injection volume was $10 \mu \mathrm{L}$ at a flow rate of $1.5 \mathrm{~mL} \mathrm{~min}^{-1}$. Samples were applied in triplicate for quantification of vinblastine. The alkaloids were quantified by using regression equation of calibration curve.

2.5. Analysis of Redox State. Endogenous $\mathrm{H}_{2} \mathrm{O}_{2}$ concentrations were determined according to Patterson et al. [28]. Hydrogen peroxide coupled with titanium sulfate generating superoxide-titanium which was a yellow precipitate. Superoxide-titanium was dissolved by sulfuric acid, and the color of the solution had a linear relation with hydrogen peroxide concentration. The absorbance was read at $415 \mathrm{~nm}$ using an ultraviolet-visible spectrophotometer (UV-2550, Shimadzu, Japan). $\mathrm{H}_{2} \mathrm{O}_{2}$ was determined from a calibration curve.

Accurately measured $0.5 \mathrm{~g}$ of $C$. roseus leaves for the determination of peroxidase activity (POD, EC 1.11.1.7) was homogenized to a fine powder under liquid nitrogen. Then, the enzyme was extracted from $5 \mathrm{~mL}$ PBS ( $\mathrm{pH}$ 7.0) with $1.0 \mathrm{mM}$ EDTA, $1.0 \mathrm{mM}$ ascorbic acid, and $10 \mathrm{~g} / \mathrm{L}$ PVP. The homogenate was centrifuged under $-4^{\circ} \mathrm{C}$ and $10000 \mathrm{~g} / \mathrm{min}$ for $30 \mathrm{~min}$. A hundred-microliter sample solution was added to $1.8 \mathrm{~mL}$ hydrochloric acid buffer solution, $1.0 \mathrm{~mL}$ guaiacol, and $0.1 \mathrm{~mL}$ hydrogen peroxide. Peroxidase activities were assayed in UV-2550 (Shimadzu, Japan) at $460 \mathrm{~nm}$ for $3 \mathrm{~min}$ [29]. 
TABLE 1: Primers used in qRT-PCR for validation of differentially expressed genes.

\begin{tabular}{lll}
\hline d4h-F & GACTTGAACTTTCATGCTGCTACAC & 25 \\
d4h-R & TCTCATCAAAAGCCTTCAATTCC & 23 \\
dat-F & AATCCCTCAGCCGCTATAACC & 21 \\
dat-R & ACGGATACGCACGTTTGGTAT & 21 \\
CrActin-F & CTATGTTCCCAGGTATTGCAGATAGA & 26 \\
CrActin-R & GCTGCTTGGAGCCAAAGC & 18 \\
CrT16H-F & GCTTCATCCACCAGTTCCAT & 20 \\
CrT16H-R & CCGGACATATCCTTCTTCCA & 20 \\
Crprx-F & GCAACATCTCCCAGACCACA & 20 \\
Crprx-R & GTTCTCCCAACACTATGAGCACC & 23 \\
ERF-F & CACCTCCAATGGCTGCTTTT & 20 \\
ERF-R & TCGCTGCCTGCTCTTCTTCT & 20 \\
\hline
\end{tabular}

2.6. RNA Extraction and RT-PCR. Total RNA was extracted from 50-100 mg samples (both leaves and roots) by TRIzol reagent and quantified by a NanoDrop ND-1000 spectrophotometer (NanoDrop Technologies) with absorbance at $260 \mathrm{~nm}$ and ethidium bromide (EB) stained test agarose gel electrophoresis used to verify the quality. cDNA was synthesized from total RNA ( $2 \mu \mathrm{g})$ using ReverTra Ace QPCR RT Kit (Toyobo, Japan) according to the manufacturer's instructions, using oligo(dT) as the primer. qRT-PCR analysis using cDNA as template and gene-specific primers was performed using a SYBR Premix Ex Taq (TaKaRa, Japan). Gene-specific primers used are listed in Table 1 (from ExPlant Technologies B.V.).

PCR was performed at $94^{\circ} \mathrm{C}$ for $5 \mathrm{~min}$ and then at $94^{\circ} \mathrm{C}$ for $30 \mathrm{~s}, 60^{\circ} \mathrm{C}$ for $30 \mathrm{~s}$, and $72^{\circ} \mathrm{C}$ for $30 \mathrm{~s}$, on $80^{\circ} \mathrm{C}$ reading plate for $1 \mathrm{~s}$ for 35 cycles, and remained at $72^{\circ} \mathrm{C}$ for $2 \mathrm{~min}$. Reactions were repeated three times for each sample to ensure the reproducibility of the results. RPS9 gene was used as an internal control. After PCR reaction, a melting curve was obtained by Opticon version 3. The comparative CT $(-\Delta \Delta \mathrm{CT})$ method was used to analyze the relative transcript levels for different experiment groups.

2.7. Statistical Analysis. Principal components analysis (PCA), the multivariate analysis tool, is used to reduce a set of original variables and to extract a small number of latent factors (principal components (PCs)) for analyzing relationships among the observed variables. PCA was performed to evaluate variations of gene expression in response to ethephon. Generally, there are three approaches used: Cattell scree test, Kaiser Criterion, and variance explained criteria. We used the scree plot method to assess the number of PCs to be retained. Loading plot, the two-dimensional plane formed by the two first principal components, is the most informative in PCA. The loading plots display the relationships among the detected compounds. The loading of PC1 and PC2 against each other shows the summary of the relationship among variables [30].

All experiments were conducted with three replicates. Statistical analysis was performed using PCA and oneway analysis of variance (ANOVA) followed by SPSS17.0. Differences between treatments were separated by the least significant difference (Duncan) test at a 0.05 probability level. The values are mean \pm SD for three samples in each group.

\section{Results}

3.1. Effect of Ethephon on Endogenous Ethylene Accumulation and Vinblastine Content. We firstly verified the effect of exogenous application of ethephon, which is absorbed into plants and converted into ethylene, on endogenous ethylene release in $C$. roseus seedling. The results showed that endogenous ethylene release was promoted with the treatment time and concentration of ethephon $(P<0.05)$ (Figure 2(a)). This indicates that the increase of vinblastine accumulation may result from the high concentration of ethephon. Then, the vinblastine contents were measured after C. roseus plants were treated by different concentrations of ethephon for $6 \mathrm{~h}$ (as is shown in Figure 2(a), we found that this is the best treatment time for $C$. roseus to produce alkaloids). Increasing concentrations of ethephon and up to $100 \mu \mathrm{M}$ ethephon concentration resulted in enhanced vinblastine content. Treatment with $100 \mu \mathrm{M}$ ethephon induced vinblastine content about 2 times that in the control group (Figure 2(b)) $(P<0.05)$.

3.2. Expression of Genes and Accumulation of Alkaloids in Response to Ethephon. The contents of vindoline and catharanthine in seedlings of $C$. roseus were also quantified. As shown in Figure 3(a), treatment with $60 \mu \mathrm{M}$ or $100 \mu \mathrm{M}$ ethephon increased the production of vindoline and catharanthine of $C$. roseus seedlings about 2 -fold, compared with the control. At the transcriptional level, the expression of $\mathrm{D} 4 \mathrm{H}$ and $\mathrm{T16H}$ genes followed a similar trend to that of vindoline (Figure 3(a)); expression of $\mathrm{D} 4 \mathrm{H}$ and $\mathrm{T} 16 \mathrm{H}$ genes was found to be 3 times more in response to high concentration of ethephon $(60 \mu \mathrm{M}$ and $100 \mu \mathrm{M})$, while the expression of $D A T$ gene was 3 times more in response to low concentration of ethephon $(45 \mu \mathrm{M})(P<0.05)$.

As indicated by the loading plot (Figure 3(c)), principal component analysis (PCA) showed two principal components, which can explain $98.181 \%$ of the variance of the four downstream TIA pathway genes and serve as high concentrations of ethephon-dependent $(\mathrm{D} 4 \mathrm{H}, \mathrm{T} 16 \mathrm{H}$, and $\mathrm{CrPr} x \mathrm{l})$ and low concentrations of ethephon-dependent $(D A T)$ variables.

\subsection{Effect of Ethephon on Redox State}

3.3.1. Effect of Ethephon on $\mathrm{H}_{2} \mathrm{O}_{2}$ Accumulation in C. roseus Leaf. The key function of plant PRXs (POD) is to oxidize phenolic substrates at the expense of ROS, mainly $\mathrm{H}_{2} \mathrm{O}_{2}$. To clearly identify the defense level of POD, the accumulation of $\mathrm{H}_{2} \mathrm{O}_{2}$ in C. roseus was also measured (Figure 4). Compared with the control plants, $\mathrm{H}_{2} \mathrm{O}_{2}$ product was inhibited by application of ethephon $(P<0.05)$. We observed that ethephon authentically reduced $\mathrm{H}_{2} \mathrm{O}_{2}$ accumulation in $C$. roseus leaf, and high concentration of ethephon significantly reduced $\mathrm{H}_{2} \mathrm{O}_{2}$ accumulation. With the treatment with $100 \mu \mathrm{M}$ ethephon, $\mathrm{H}_{2} \mathrm{O}_{2}$ product was 2-fold less in 
TABLE 2: The relative transcript level of CrPrxl and the correlation with vinblastine and POD activityafter 12 or $24 \mathrm{~h}$ of treatment with ethephon to C. roseus. The results shown are the means of three replicates; bars represent SE.

\begin{tabular}{ccccccc}
\hline & $\mathrm{CK}$ & $+45 \mu \mathrm{M}$ & $+60 \mu \mathrm{M}$ & $+100 \mu \mathrm{M}$ & Vinblastine & POD activity \\
\hline $12 \mathrm{~h}$ & 1 & $2.181 \pm 0.021^{\mathrm{a}}$ & $2.266 \pm 0.042^{\mathrm{a}}$ & $3.249 \pm 0.148^{\mathrm{b}}$ & $0.918^{* *}$ & $0.940^{* *}$ \\
\hline $24 \mathrm{~h}$ & $1.765 \pm 0.005^{\mathrm{a}}$ & $2.354 \pm 0.019^{\mathrm{b}}$ & $3.434 \pm 0.040^{\mathrm{c}}$ & $6.714 \pm 0.146^{\mathrm{d}}$ & $0.816^{* *}$ & $0.951^{* *}$ \\
\hline
\end{tabular}

Different letters indicate significant differences among treatments $(P<0.05) .{ }^{* *}$ Correlation is significant at the 0.01 level.

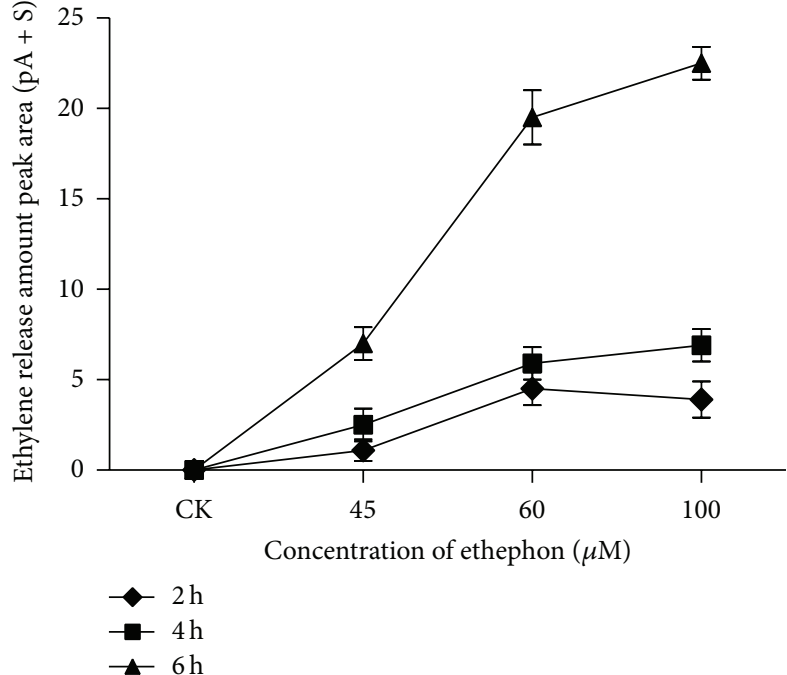

(a)

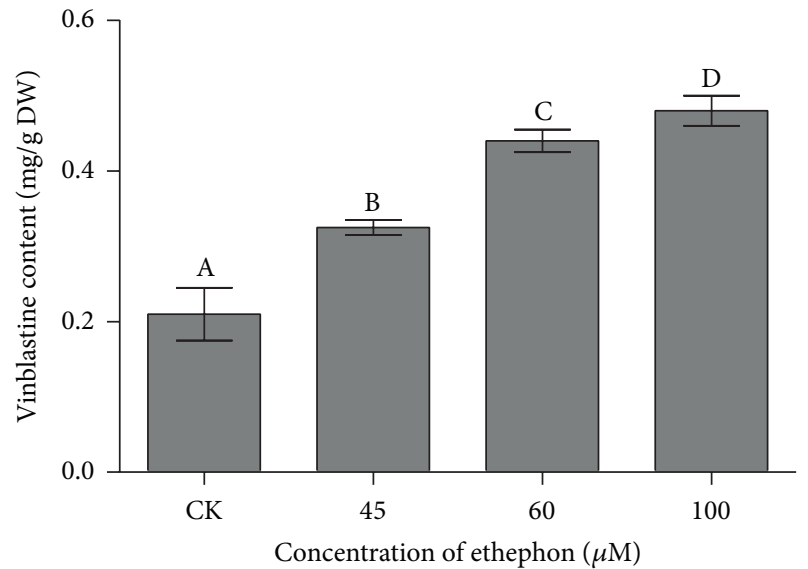

(b)

Figure 2: The effect of different concentrations of ethephon on endogenous (a) ethylene release amount and (b) vinblastine contents in Catharanthus roseus. The results shown are the means of three replicates; bars represent SE. Different letters indicate significant differences among treatments $(P<0.05)$.

comparison with contrast $(P<0.05)$. This suggested that POD activity decreased $\mathrm{H}_{2} \mathrm{O}_{2}$ accumulation in $C$. roseus leaf.

3.3.2. Effect of Ethephon on Peroxidase Activity. In C. roseus, POD was shown to be responsible for the dimerization reaction between catharanthine and vindoline to produce $\alpha-3^{\prime}, 4^{\prime}$ anhydrovinblastine, the precursor of the natural antitumoral products, vinblastine and vincristine. To determine the effect of ethephon on peroxidase (POD) of C. roseus, different ethephon concentrations were added to the media (Figure 5). It is noticed that POD activity had a lasting upward trend and showed peak of maximal activity in the seedlings treated by $100 \mu \mathrm{M}$ ethephon $(P<0.05)$.

3.4. Effect of Ethephon on CrPrx1 Transcript. The transcript level of CrPrxl in C. roseus including control and treated by ethephon at $0 \mathrm{~h}, 12 \mathrm{~h}$, and $24 \mathrm{~h}$ was quantified (Table 2). In comparison to vinblastine content, the exposure of seedlings to ethephon led to a positive effect on the transcription of CrPrx1 $(P<0.05)$. Responding to these concentrations of ethephon, additive transcription of $\operatorname{CrPrx1}$ was observed obviously at both $12 \mathrm{~h}$ and $24 \mathrm{~h}$ and the increment was prominent at $100 \mu \mathrm{M}$ ethephon. Moreover, the $\operatorname{Cr} \operatorname{Prx} 1$ transcript level at $24 \mathrm{~h}$ was about 2 times higher than at $12 \mathrm{~h}$; particularly, maximal steady-state amounts of CrPrxl transcripts were detected in seedlings treated by $100 \mu \mathrm{M}$ ethephon.
3.5. The Loading Plot Combination with Correlation Analysis. We also analyzed the correlation between alkaloids and the transcript levels of genes (the downstream TIA pathway genes and ethylene responsive factor $(E R F)$ gene) (Figure 6). As indicated by loading plot (Figure 6), there were two principal components, which can explain $84.164 \%$ of the variance of the ten factors and serve as the first principle component $(\mathrm{D} 4 \mathrm{H}$, vinblastine, vindoline, catharanthine, and $\mathrm{H}_{2} \mathrm{O}_{2}$ ) and the second principle component (DAT, T16H, CrPrxl, ERF, and POD) variables. $\mathrm{H}_{2} \mathrm{O}_{2}$ presented a significant level of negative correlation in vinblastine accumulation during ethephon treatments, while ERF presented a significant level of activity in TIAs accumulation during ethephon treatments. In addition, with the loading plot combination with correlation analysis (Table 2), the results revealed that $\operatorname{CrPrx1}$ transcript was significantly correlated with vinblastine content and POD activity $(P<0.01)$.

\section{Discussion}

From the present study, it can be concluded that vinblastine biosynthesis influenced by ethephon has a specific relationship with the downstream TIA pathway genes. The result revealed that exogenous ethephon induced a slight increase in endogenous ethylene synthesis (Figure 2(a)). A significant amount of evidence revealed that the regulator had a close 


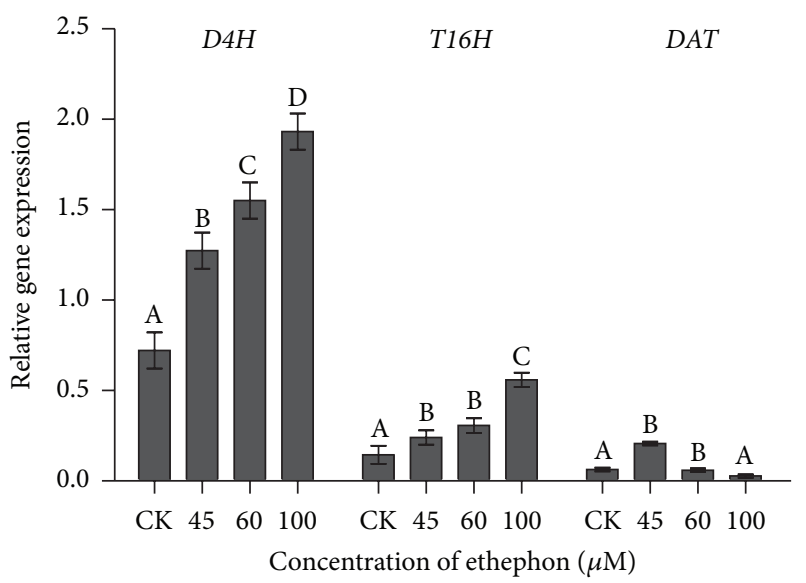

(a)

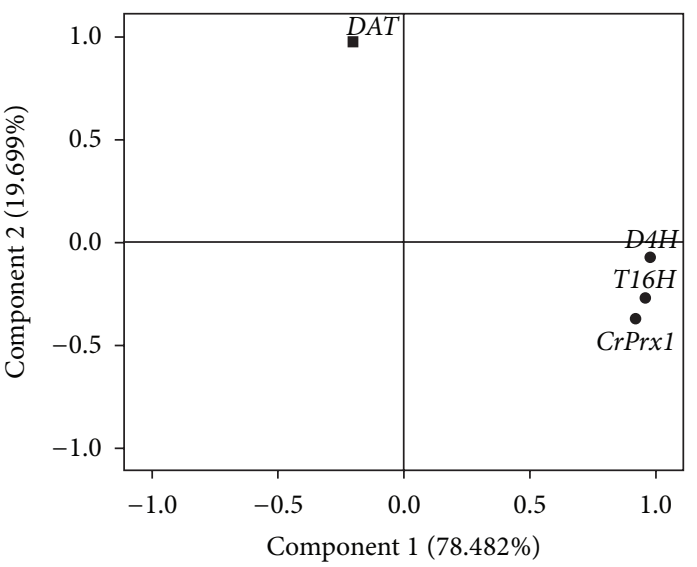

(b)

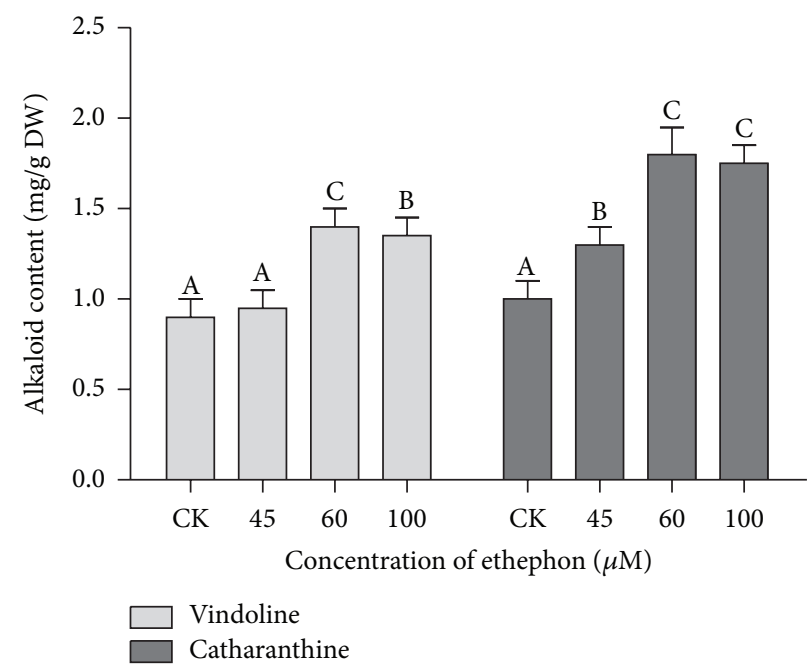

(c)

FIGURE 3: Expression of genes and accumulation of alkaloids in response to ethephon. (a) Relative expression of $D A T, D 4 H$, and T16H genes in Catharanthus roseus. (b) Loading plot of PCA. The first principal component (PC1) was the expression of genes D4H, T16H, and CrPrxl, which were shown as $\bullet$. The second principal component (PC2) was the expression of gene DAT, which was shown as $\mathbf{m}$. (c) Vindoline and catharanthine contents in Catharanthus roseus. The results shown are the means of three replicates; bars represent SE. Different letters indicate significant differences among treatments $(P<0.05)$.

relationship with plant growth or secondary metabolism [31]. Tadiello et al. found that all plant tissues are able to produce ethylene, which has been established to modulate a number of important plant physiological activities [32]. The use of elicitors to promote secondary metabolism in plant cell and tissue cultures has become a common practice [33]. Therefore, it is not surprising that ethephon which is converted into ethylene upon metabolism by plants influenced vinblastine accumulation in C. roseus (Figure 2(b)). We noticed that vinblastine content was gradually increasing and exceeded the control group at $45 \sim 100 \mu \mathrm{M}$ ethephon. The level of CrPrx1 transcript is similar as a trend of vinblastine content of $C$. roseus treated by ethephon (Table 2). Furthermore, through analyzing the correlation between vinblastine and transcription of the downstream TIA pathway genes (Figure 6), we found that the results have significant correlation with each other. As the common precursor of all dimeric alkaloids, $\alpha-3^{\prime}, 4^{\prime}$ anhydrovinblastine (AVLB) was converted into vinblastine through coupling of the monomeric precursors vindoline and catharanthine [7]. Peroxidase was proposed to be a channeling mechanism for the peroxidase mediated-vacuolar synthesis of AVLB $[4,6]$. Our data also indirectly indicated that, on the molecular level, vinblastine biosynthesis had a close correlation with peroxidase.

POD is the key enzyme catalyzing vinblastine production. The data revealed that POD activity depending on CrPrx1 transcript was assuredly promoted by ethephon. From the result of the three alkaloids and POD activity (Figures 2(b), 3, and 5), it was shown that vinblastine content was more sensitive to the activity of peroxidase; therefore, POD activity played a principal role in vinblastine synthesis. Under $60 \mu \mathrm{M}$ ethephon treatment, vindoline and catharanthine 


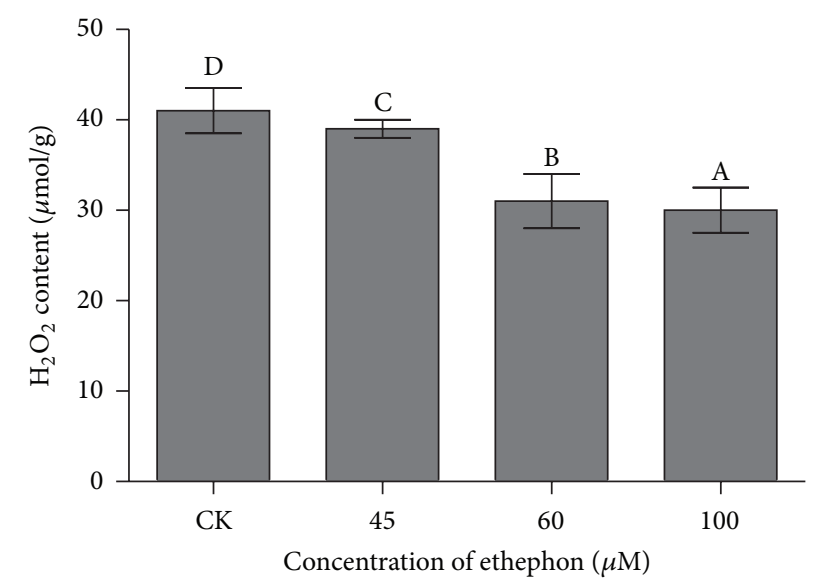

Figure 4: The effects of different concentrations of ethephon on $\mathrm{H}_{2} \mathrm{O}_{2}$ accumulation in Catharanthus roseus. The results shown are the means of three replicates; bars represent SE. Different letters indicate significant differences among treatments $(P<0.05)$.

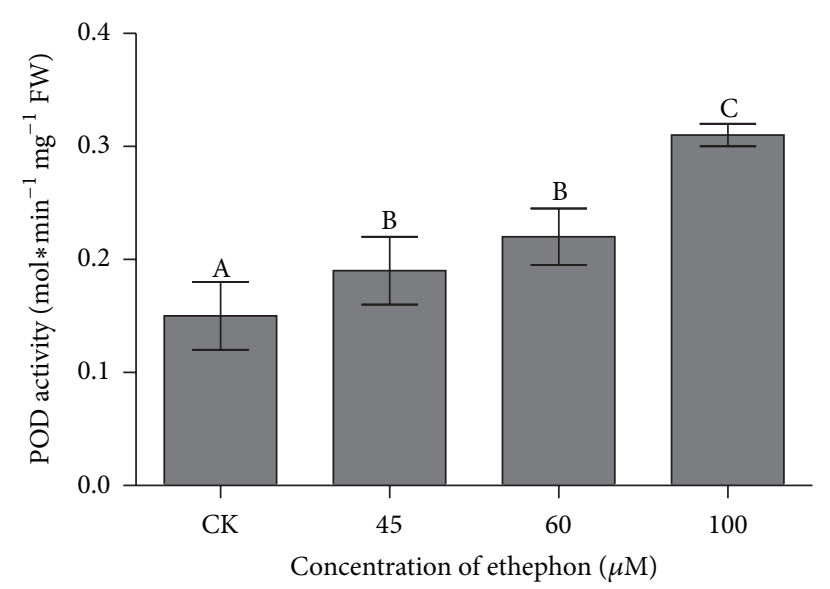

FIGURE 5: The effects of different concentrations of ethephon on peroxidase activity in leaves of Catharanthus roseus. The results shown are the means of three replicates; bars represent SE. Different letters indicate significant differences among treatments $(P<0.05)$.

contents were a little higher than that of $100 \mu \mathrm{M}$ ethephon, but vinblastine content was decreased possibly leading to lower POD activity. We analyzed the correlation between POD activity and transcription of CrPrxl (Table 2), and the results also had significant correlation. POD and CrPrxl are small part of a complex network of factors subjected to different regulation programs in the TIA pathway and this pathway is not only a single enzyme matching the regulation [3]. Researchers enabled the observation of a clear correlation between the AVLB and CrPrxl transcript levels, but details of the process where $\operatorname{CrPrx} 1$ regulated vinblastine biosynthesis are not clear. In addition, POD, dependent on CrPrx1 transcript, has defense and catalyzing vinblastine biosynthesis mechanism; thus, it plays a more complex role in the process. Our results demonstrate that ethylene regulates vinblastine accumulation via controlling $\operatorname{CrPrxl}$ transcript and protein which catalyze precursor of vinblastine synthesis

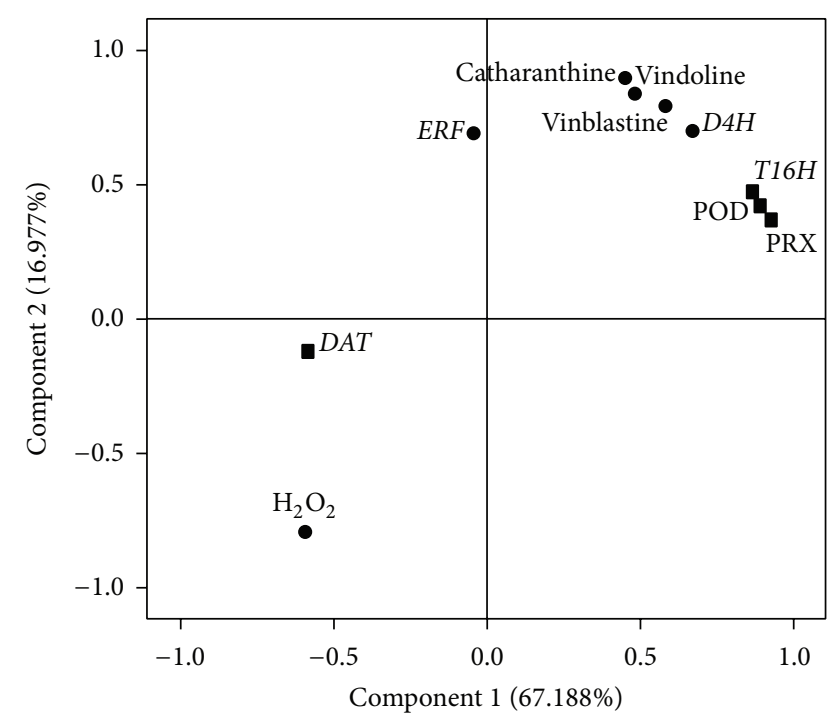

FIGURE 6: Loading plot of PCA. The first principal component (PC1) was the expression of gene $\mathrm{D} 4 \mathrm{H}$ and the content of vinblastine, vindoline, and catharanthine and the activity of $\mathrm{H}_{2} \mathrm{O}_{2}$, which were shown as $\bullet$. The second principal component (PC2) was the expression of genes DAT, CrPrxl, T16H, and ERF and the activity of POD, which were shown as $\boldsymbol{m}$. The results shown are the means of three replicates; bars represent SE.

at the molecular level. The spatial, temporal, and inducible formation of secondary metabolites and the transcripts of corresponding biosynthetic genes are under tight regulation at different levels, in which transcriptional regulation via transcription factors has been investigated intensively [31]. Guo et al. found that activation of EIN3 (ethylene insensitive 3) increased peroxidase (POD) activity through the direct transcriptional regulation of PODs expression. Accordingly, ethylene pretreatment or EIN3 activation was able to preclude excess ROS accumulation and increased tolerance to salt stress [34]. In fact, not all regulation is at the level of the EIN3-like transcription factors, and downstream ERF and EDF transcription factors greatly increase the potential points for interaction and cross talk of ethylene signaling with other pathways [23]. Therefore, we tested the expression of ethylene responsive factor (ERF) and investigated the possible regulatory way, by which ethylene controls gene transcription. As shown in Figure 6, the ERF mainly participates in the accumulation of TIAs in response to ethephon treatment. This suggests that $E R F$ may be the central hub of the POD and CrPrxl and, thus, may be actively involved in accumulation of TIAs in response to ethephon.

In vitro, a range of secondary metabolites including phenols, amines, indoles, alkaloids, and sulphonates may act as reductant substrates of class III peroxidases (Prxs), at the expense of $\mathrm{H}_{2} \mathrm{O}_{2}$, which has also emerged as a pivotal molecule in the responses of a range of biotic and abiotic stresses [35]. In this work, we showed increased accumulation of vinblastine, vindoline, and catharanthine with treatment with ethephon. Meanwhile, transcription of the peroxidase (POD) encoding gene CrPrxl is also increased by 
ethephon treatment, accompanied by increased POD activity and decreased $\mathrm{H}_{2} \mathrm{O}_{2}$ content. POD is generally considered to be merely a peroxide-detoxifying enzyme, especially $\mathrm{H}_{2} \mathrm{O}_{2}$ [8]. Hence, $\mathrm{H}_{2} \mathrm{O}_{2}$ accumulation is the evidence to indirectly prove POD and vinblastine synthesis conditions. If this inference is established, $\mathrm{H}_{2} \mathrm{O}_{2}$ accumulation must conform to the trend of POD activity level. According to the result of $\mathrm{H}_{2} \mathrm{O}_{2}$ accumulation, $\mathrm{H}_{2} \mathrm{O}_{2}$ content was reduced by ethephon; therefore, the result consisted with the above reasoning. Some research indicated that $\mathrm{H}_{2} \mathrm{O}_{2}$ content was correlated with vinblastine accumulation [36, 37].

The role of ethylene in plant defense is controversial as it contributes to resistance in some interactions [38, 39] but promotes disease in others [40-42]. Therefore, we suppose that vinblastine may be suppressed by trace of ethephon, while being promoted by higher concentration of ethephon. It should be noted that this study does not provide enough data to understand the behavior of ethylene in these systems [5]. Ethylene interacts with other plant developmental pathways and the mechanisms must be studied in order to clarify these issues in more detail [23].

$\begin{array}{ll}\text { Abbreviations } \\ \text { AVLB: } & \alpha-3^{\prime}, 4^{\prime} \text {-Anhydrovinblastine } \\ \text { CrPrxl: } & \text { Catharanthus roseus peroxidase } \\ \text { DAT: } & \text { Acetyl-CoA: } \\ & \text { 4-O-deacetylvindoline-4-O-acetyl-transferase } \\ \text { DEPC: } & \text { Diethylpyrocarbonate } \\ \text { D4H: } & \text { Desacetoxyvindoline-4-hydroxylase } \\ \text { EDTA: } & \text { Ethylenediaminetetraacetic acid } \\ \text { ERF: } & \text { Ethylene response factor } \\ \text { MMLV: } & \text { Moloney murine leukemia virus } \\ \text { POD: } & \text { Peroxidase } \\ \text { RT-PCR: } & \text { Reverse transcription-polymerase chain } \\ & \text { reaction } \\ \text { TIAs: } & \text { Terpenoid indole alkaloids } \\ \text { T16H: } & \text { Tabersonine-16-hydroxylase } \\ \text { PCA: } & \text { Principal component analysis } \\ \text { PVP: } & \text { Polyvinyl pyrrolidone. }\end{array}$

\section{Competing Interests}

The authors declare that there are no competing interests regarding the publication of this paper.

\section{Authors' Contributions}

Xiao-Rui Guo and Zhong-Hua Tang designed and supported the work. Xi Wang and Bo-Wen Chang conducted the experiments and prepared the paper. Ya-Jie Pan conducted the supplementary experiments and revised the paper. YanBo $\mathrm{Hu}$ edited the paper and Xiao-Rui Guo and ZhongHua Tang reviewed and revised it. Xi Wang and Ya-Jie Pan contributed equally to this work.

\section{Acknowledgments}

This study was financially supported by the Fundamental Research Funds for the Central Universities (2572014DA01) and the Natural Science Foundation of Heilongjiang province (C201117).

\section{References}

[1] M. M. R. Costa, F. Hilliou, P. Duarte et al., "Molecular cloning and characterization of a vacuolar class III peroxidase involved in the metabolism of anticancer alkaloids in Catharanthus roseus," Plant Physiology, vol. 146, no. 2, pp. 403-417, 2008.

[2] Z. H. Tang, Y. J. Liu, X. R. Guo, and Y. G. Zu, “The combined effects of salinity and nitrogen forms on Catharanthus roseus: the role of internal ammonium and free amino acids during salt stress," Journal of Plant Nutrition and Soil Science, vol. 174, no. 1, pp. 135-144, 2011.

[3] B. St-Pierre, F. A. Vazquez-Flota, and V. De Luca, "Multicellular compartmentation of Catharanthus roseus alkaloid biosynthesis predicts intercellular translocation of a pathway intermediate," Plant Cell, vol. 11, no. 5, pp. 887-900, 1999.

[4] M. Sottomayor, M. López-Serrano, F. DiCosmo, and A. Ros Barceló, "Purification and characterization of $\alpha-3,4$ 'anhydrovinblastine synthase (peroxidase-like) from Catharanthus roseus (L.) G. Don," FEBS Letters, vol. 428, no. 3, pp. 299303, 1998.

[5] L. Almagro, L. V. Gómez Ros, S. Belchi-Navarro, R. Bru, A. Ros Barceló, and M. A. Pedreño, "Class III peroxidases in plant defence reactions," Journal of Experimental Botany, vol. 60, no. 2, pp. 377-390, 2009.

[6] M. Sottomayor, I. Lopes Cardoso, L. G. Pereira, and A. Ros Barceló, "Peroxidase and the biosynthesis of terpenoid indole alkaloids in the medicinal plant Catharanthus roseus (L.) G. Don," Phytochemistry Reviews, vol. 3, no. 1-2, pp. 159-171, 2004.

[7] R. Verpoorte, R. van der Heijden, and P. R. H. Moreno, "Chapter 3: biosynthesis of terpenoid indole alkaloids in Catharanthus roseus cells," in The Alkaloids: Chemistry and Pharmacology, pp. 221-299, Academic Press, New York, NY, USA, 1997.

[8] T. Kawano, "Roles of the reactive oxygen species-generating peroxidase reactions in plant defense and growth induction," Plant Cell Reports, vol. 21, no. 9, pp. 829-837, 2003.

[9] H. Yamasaki, Y. Sakihama, and N. Ikehara, "Flavonoidperoxidase reaction as a detoxification mechanism of plant cells against $\mathrm{H}_{2} \mathrm{O}_{2}$," Plant Physiology, vol. 115, no. 4, pp. 1405-1412, 1997.

[10] F. Passardi, M. Tognolli, M. De Meyer, C. Penel, and C. Dunand, "Two cell wall associated peroxidases from Arabidopsis influence root elongation," Planta, vol. 223, no. 5, pp. 965-974, 2006.

[11] F. Passardi, C. Cosio, C. Penel, and C. Dunand, "Peroxidases have more functions than a Swiss army knife," Plant Cell Reports, vol. 24, no. 5, pp. 255-265, 2005.

[12] J. Díaz, F. Pomar, Á. Bernal, and F. Merino, "Peroxidases and the metabolism of capsaicin in Capsicum annuum L.," Phytochemistry Reviews, vol. 3, no. 1-2, pp. 141-157, 2004.

[13] B. K. Kristensen, K. Burhenne, and S. K. Rasmussen, "Peroxidases and the metabolism of hydroxycinnamic acid amides in Poaceae," Phytochemistry Reviews, vol. 3, no. 1-2, pp. 127-140, 2004. 
[14] A. Mika, F. Minibayeva, R. Beckett, and S. Lüthje, "Possible functions of extracellular peroxidases in stress-induced generation and detoxification of active oxygen species," Phytochemistry Reviews, vol. 3, no. 1-2, pp. 173-193, 2004.

[15] S. Sang, C. S. Yang, and C.-T. Ho, "Peroxidase-mediated oxidation of catechins," Phytochemistry Reviews, vol. 3, no. 1-2, pp. 229-241, 2004.

[16] K. Yoshida, P. Kaothien, T. Matsui, A. Kawaoka, and A. Shinmyo, "Molecular biology and application of plant peroxidase genes," Applied Microbiology and Biotechnology, vol. 60, no. 6, pp. 665-670, 2003.

[17] B. Chang, L. Yang, W. Cong, Y. Zu, and Z. Tang, “The improved resistance to high salinity induced by trehalose is associated with ionic regulation and osmotic adjustment in Catharanthus roseus," Plant Physiology and Biochemistry, vol. 77, pp. 140-148, 2014.

[18] X.-R. Guo, B.-W. Chang, Y.-G. Zu, and Z.-H. Tang, "The impacts of increased nitrate supply on Catharanthus roseus growth and alkaloid accumulations under ultraviolet-B stress," Journal of Plant Interactions, vol. 9, no. 1, pp. 640-646, 2014.

[19] B.-W. Chang, W.-W. Cong, Q. Chen, Y.-G. Zu, and Z.-H. Tang, "The influence of different forms and concentrations of potassium nutrition on growth and alkaloid metabolism in Catharanthus roseus seedlings," Journal of Plant Interactions, vol. 9, no. 1, pp. 370-377, 2014.

[20] X.-R. Guo, Y.-G. Zu, and Z.-H. Tang, "Physiological responses of Catharanthus roseus to different nitrogen forms," Acta Physiologiae Plantarum, vol. 34, no. 2, pp. 589-598, 2012.

[21] T. M. Kutchan, "Ecological arsenal and developmental dispatcher. The paradigm of secondary metabolism," Plant Physiology, vol. 125, no. 1, pp. 58-60, 2001.

[22] Y. Lin, L. Yang, M. Paul, Y. Zu, and Z. Tang, "Ethylene promotes germination of Arabidopsis seed under salinity by decreasing reactive oxygen species: evidence for the involvement of nitric oxide simulated by sodium nitroprusside," Plant Physiology and Biochemistry, vol. 73, pp. 211-218, 2013.

[23] G. E. Schaller, "Ethylene and the regulation of plant development," BMC Biology, vol. 10, article 9, 2012.

[24] S. Zhong, H. Shi, C. Xue et al., "A molecular framework of light-controlled phytohormone action in arabidopsis," Current Biology, vol. 22, no. 16, pp. 1530-1535, 2012.

[25] K. Sasaki, O. Yuichi, S. Hiraga et al., "Characterization of two rice peroxidase promoters that respond to blast fungusinfection," Molecular Genetics and Genomics, vol. 278, no. 6, pp. 709-722, 2007.

[26] I. T. Baldwin, Z.-P. Zhang, N. Diab et al., "Quantification, correlations and manipulations of wound-induced changes in jasmonic acid and nicotine in Nicotiana sylvestris," Planta, vol. 201, no. 4, pp. 397-404, 1997.

[27] N. Hibi, S. Higashiguchi, T. Hashimoto, and Y. Yamada, "Gene expression in tobacco low-nicotine mutants," Plant Cell, vol. 6, no. 5, pp. 723-735, 1994.

[28] B. D. Patterson, E. A. MacRae, and I. B. Ferguson, "Estimation of hydrogen peroxide in plant extracts using titanium(IV)," Analytical Biochemistry, vol. 139, no. 2, pp. 487-492, 1984.

[29] M. Jiang and J. Zhang, "Water stress-induced abscisic acid accumulation triggers the increased generation of reactive oxygen species and up-regulates the activities of antioxidant enzymes in maize leaves," Journal of Experimental Botany, vol. 53, no. 379, pp. 2401-2410, 2002.
[30] Y.-J. Pan, J. Liu, X.-R. Guo, Y.-G. Zu, and Z.-H. Tang, “Gene transcript profiles of the TIA biosynthetic pathway in response to ethylene and copper reveal their interactive role in modulating TIA biosynthesis in Catharanthus roseus," Protoplasma, vol. 252, no. 3, pp. 813-824, 2015.

[31] C.-Q. Yang, X. Fang, X.-M. Wu, Y.-B. Mao, L.-J. Wang, and X.-Y. Chen, "Transcriptional regulation of plant secondary metabolism," Journal of Integrative Plant Biology, vol. 54, no. 10, pp. 703-712, 2012.

[32] A. Tadiello, V. Ziosi, A. S. Negri et al., "On the role of ethylene, auxin and a GOLVEN-like peptide hormone in the regulation of peach ripening," BMC Plant Biology, vol. 16, no. 1, article 44, 2016.

[33] F. Vázquez-Flota, E. Hernández-Domínguez, M. De Lourdes Miranda-Ham, and M. Monforte-González, "A differential response to chemical elicitors in Catharanthus roseus in vitro cultures," Biotechnology Letters, vol. 31, no. 4, pp. 591-595, 2009.

[34] J. Peng, Z. Li, X. Wen et al., "Salt-induced stabilization of EIN3/EIL1 confers salinity tolerance by deterring ROS accumulation in Arabidopsis," PLoS Genetics, vol. 10, no. 10, Article ID e1004664, 2014.

[35] F. Ferreres, R. Figueiredo, S. Bettencourt et al., "Identification of phenolic compounds in isolated vacuoles of the medicinal plant Catharanthus roseus and their interaction with vacuolar class III peroxidase: an $\mathrm{H}_{2} \mathrm{O}_{2}$ affair?" Journal of Experimental Botany, vol. 62, no. 8, pp. 2841-2854, 2011.

[36] M. Jaggi, S. Kumar, and A. K. Sinha, "Overexpression of an apoplastic peroxidase gene CrPrx in transgenic hairy root lines of Catharanthus roseus," Applied Microbiology and Biotechnology, vol. 90, no. 3, pp. 1005-1016, 2011.

[37] Z. Tang, L. Yang, Y. Zu, and X. Guo, "Variations of vinblastine accumulation and redox state affected by exogenous $\mathrm{H}_{2} \mathrm{O}_{2}$ in Catharanthus roseus (L.) G. Don," Plant Growth Regulation, vol. 57, no. 1, pp. 15-20, 2009.

[38] C. Norman-Setterblad, S. Vidal, and E. T. Palva, "Interacting signal pathways control defense gene expression in Arabidopsis in response to cell wall-degrading enzymes from Erwinia carotovora," Molecular Plant-Microbe Interactions, vol. 13, no. 4, pp. 430-438, 2000.

[39] B. P. H. J. Thomma, K. Eggermont, K. F. M.-J. Tierens, and W. F. Broekaert, "Requirement of functional ethylene-insensitive 2 gene for efficient resistance of Arabidopsis to infection by Botrytis cinerea," Plant Physiology, vol. 121, no. 4, pp. 1093-1101, 1999.

[40] T. Hoffman, J. S. Schmidt, X. Zheng, and A. F. Bent, "Isolation of ethylene-insensitive soybean mutants that are altered in pathogen susceptibility and gene-for-gene disease resistance," Plant Physiology, vol. 119, no. 3, pp. 935-949, 1999.

[41] S. T. Lund, R. E. Stall, and H. J. Klee, "Ethylene regulates the susceptible response to pathogen infection in tomato," Plant Cell, vol. 10, no. 3, pp. 371-382, 1998.

[42] A. F. Bent, R. W. Innes, J. R. Ecker, and B. J. Staskawicz, "Disease development in ethylene-insensitive Arabidopsis thaliana infected with virulent and avirulent Pseudomonas and Xanthomonas pathogens," Molecular Plant-Microbe Interactions, vol. 5, no. 5, pp. 372-378, 1992. 

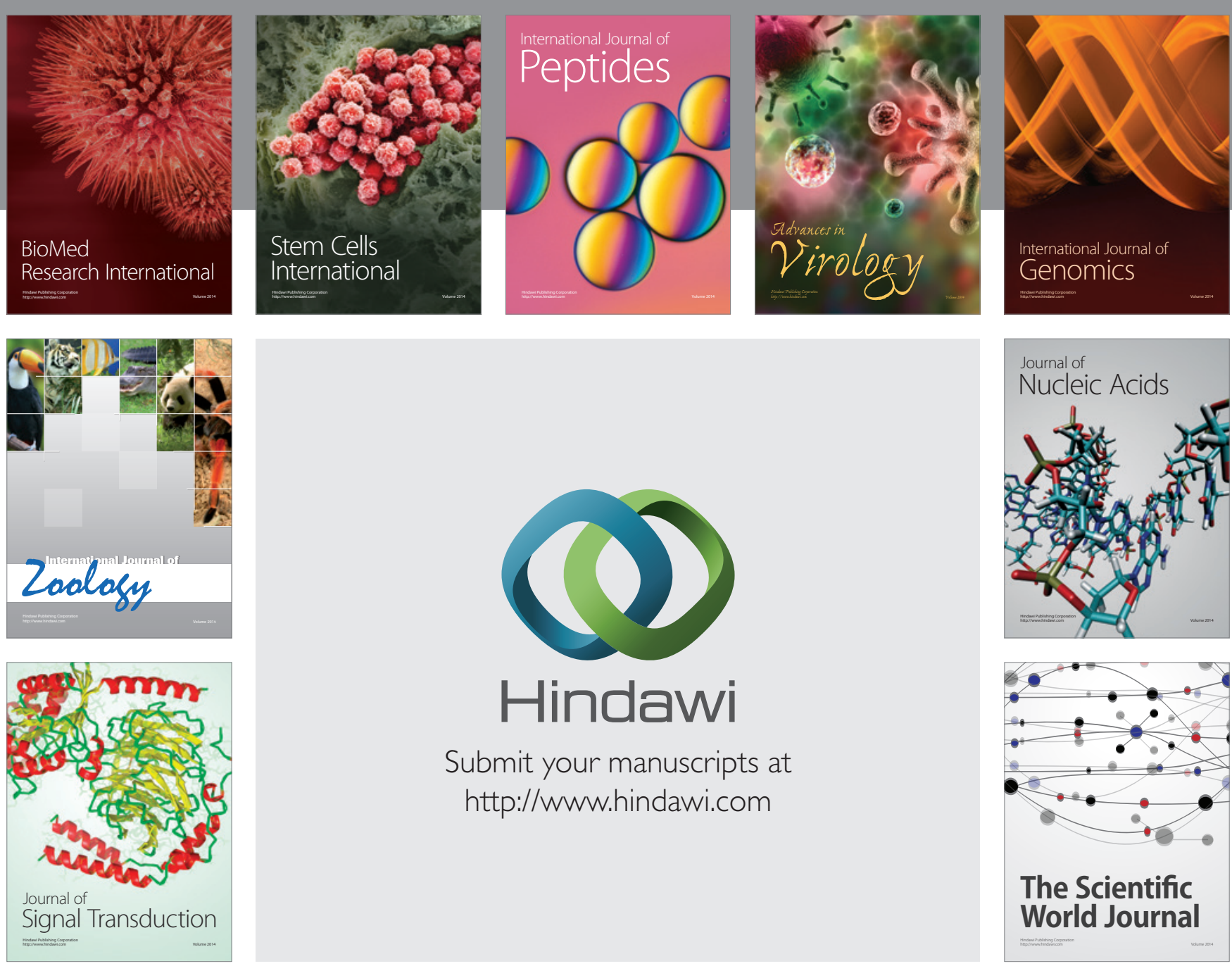

Submit your manuscripts at

http://www.hindawi.com
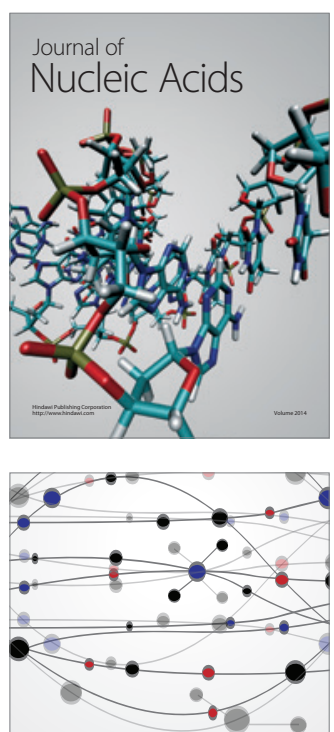

The Scientific World Journal
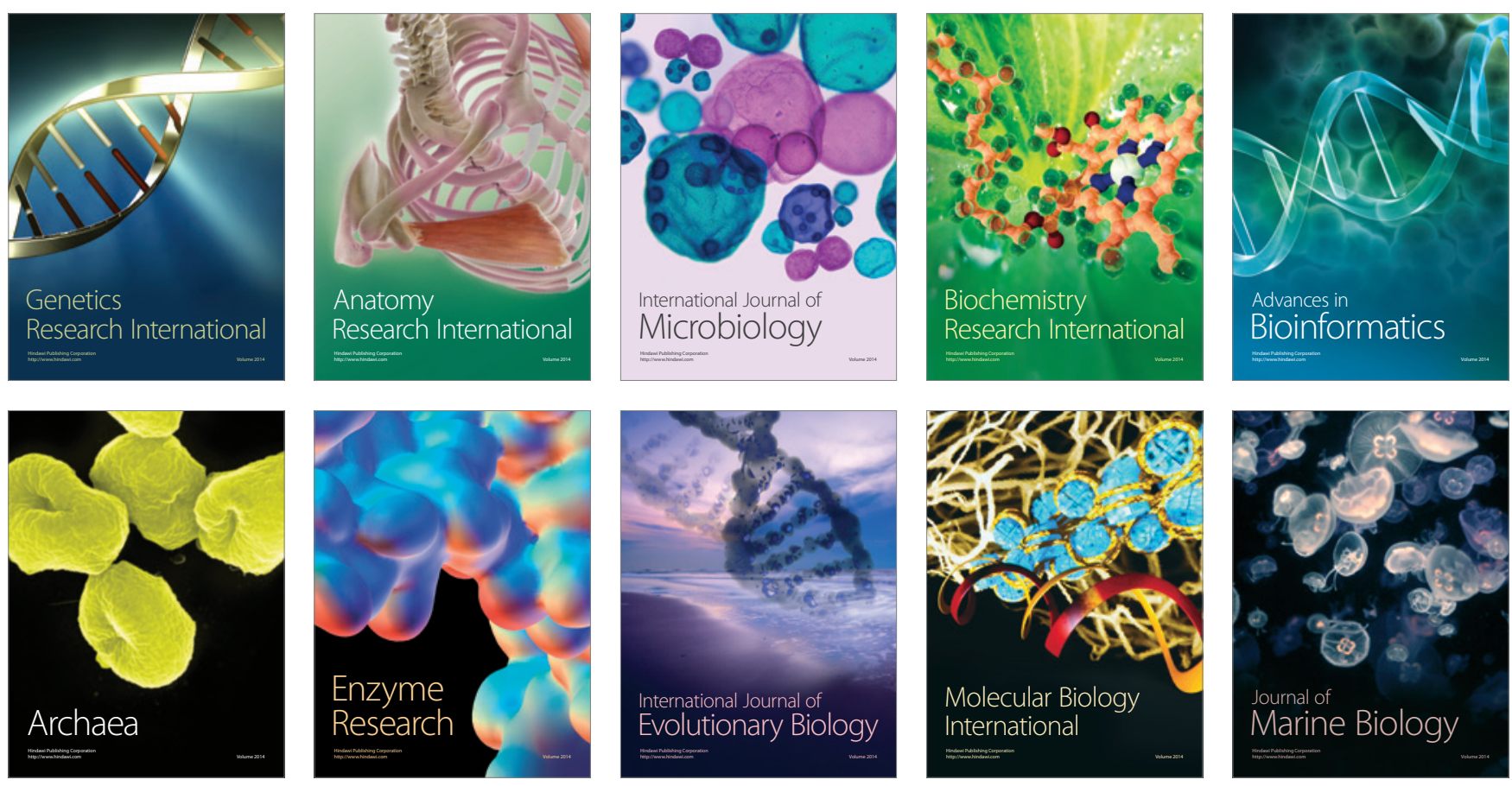